\title{
Genetic Variations Leading to Familial Dilated Cardiomyopathy
}

\author{
Kae Won Cho', Jongsung Lee ${ }^{2}$, and Youngjo Kim ${ }^{1, *}$
}

Cardiomyopathy is a major cause of death worldwide. Based on pathohistological abnormalities and clinical manifestation, cardiomyopathies are categorized into several groups: hypertrophic, dilated, restricted, arrhythmogenic right ventricular, and unclassified. Dilated cardiomyopathy, which is characterized by dilation of the left ventricle and systolic dysfunction, is the most severe and prevalent form of cardiomyopathy and usually requires heart transplantation. Its etiology remains unclear. Recent genetic studies of single gene mutations have provided significant insights into the complex processes of cardiac dysfunction. To date, over $\mathbf{4 0}$ genes have been demonstrated to contribute to dilated cardiomyopathy. With advances in genetic screening techniques, novel genes associated with this disease are continuously being identified. The respective gene products can be classified into several functional groups such as sarcomere proteins, structural proteins, ion channels, and nuclear envelope proteins. Nuclear envelope proteins are emerging as potential molecular targets in dilated cardiomyopathy. Because they are not directly associated with contractile force generation and transmission, the molecular pathways through which these proteins cause cardiac muscle disorder remain unclear. However, nuclear envelope proteins are involved in many essential cellular processes. Therefore, integrating apparently distinct cellular processes is of great interest in elucidating the etiology of dilated cardiomyopathy. In this mini review, we summarize the genetic factors associated with dilated cardiomyopathy and discuss their cellular functions.

\section{INTRODUCTION}

Cardiomyopathy is a main cause of death in most developed

\footnotetext{
${ }^{1}$ Soonchunhyang Institute of Medi-bio Science, Soonchunhyang University, Cheon-an 31151, Korea, '2Department of Genetic Engineering, College of Biotechnology and Bioengineering, Sungkyunkwan University, Suwon 16419, Korea

${ }^{*}$ Correspondence: yjokim@sch.ac.kr
}

Received 14 March, 2016; revised 21 October, 2016; accepted 28 October, 2016; published online 31 October, 2016

Keywords: dilated cardiomyopathy, missense mutation, nuclear envelope, sarcomere protein, structural protein countries (Mortality and Causes of Death, 2015). According to the American Heart Association's definition, cardiomyopathy is a group of diseases of the myocardium characterized by improper ventricular hypertrophy or dilation, and lead to mechanical and electrical dysfunction of the heart (Maron et al., 2006). Based on morphological abnormalities and clinical manifestations, cardiomyopathies are classified as hypertrophic cardiomyopathy (HCM), dilated cardiomyopathy (DCM), arrhythmogenic right ventricular cardiomyopathy (ARVC), restrictive cardiomyopathy (RCM), LV noncompaction, conduction system disease, ion channelopathies, myocarditis (inflammatory cardiomyopathy), or stress cardiomyopathy.

Patients with DCM typically exhibit enlargement of the left ventricular chamber and often experience systolic abnormalities. DCM is the third leading cause of heart failure and frequently requires heart transplantation (Maron et al., 2006). The estimated prevalence of DCM is 1:2,500-3,000 (Codd et al., 1989; Miura et al., 2002). Moreover, approximately $20-35 \%$ of DCMs is familial and is associated with at least 40 genes or loci. With advances in genetic technologies used for diagnosis of DCMs, the number of genes proposed to contribute to cardiomyopathy has increased to at least 100 (Dellefave and McNally, 2010). Genetic alterations detected in patients with familial DCM are usually inherited in a dominant-negative manner. Although only a subset of DCM is caused by specific gene mutations, studies of these gene mutations and their phenotypes will provide molecular insights into the etiology of DCM.

Familial DCM was once considered a single specific disease; however, it is now known to have heterogeneous histopathological features and penetrance according to the causative gene mutations. Genes associated with DCMs are classified into several groups, including genes encoding nuclear envelope proteins, sarcomere proteins, structural proteins, ion channels, and unclassified proteins (Dellefave and McNally, 2010). In this review, we will discuss known genetic causes and cellular processes underlying DCM.

\section{MUTATIONS IN SARCOMERE PROTEINS LEADING TO DCM}

Myofibril consists of a basic unit called the sarcomere (Fig. 1) Contractile force generation in cardiomyocytes and its faithful delivery to the extracellular matrix and neighboring cells are essential for heart function. Over 300 mutations in sarcomere proteins have been shown as causes of DCM and other heart diseases (Morita et al., 2005). Human genetic studies have shown that many familial DCM cases are linked to alterations in 


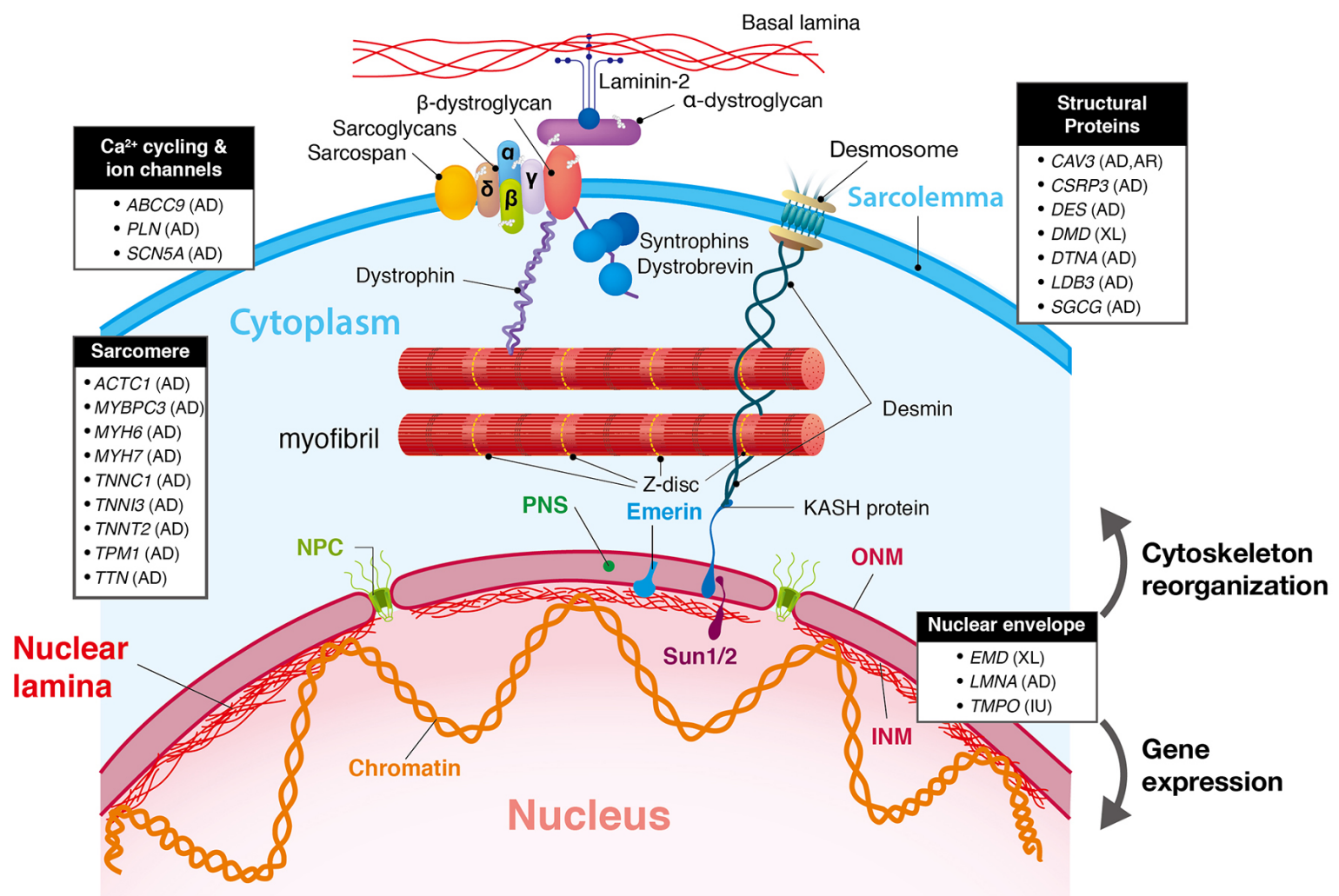

Fig. 1. Functional groups of genes associated with dilated cardiomyopathy. Genes known to lead to dilated cardiomyopathy indicated in the boxes. Myofibrils in cardiomyocytes are connected to the cytoplasmic and nuclear membranes through filamentous systems. Actin filaments are directly linked to dystrophin, which is connected to dystrophic-associated protein complex. The dystrophin-associated protein complex is connected to the extracellular matrix through laminin. Desmin anchors myofibrils to both the cytoplasmic membrane and the nuclear envelope. Desmin comes in contact with desmosome proteins at the cytoplasmic membrane. Myofibril is surrounded by the desmin filament system, and the nuclear envelope is connected with the contractile machinery through interactions between desmin and KASH domain proteins. The nuclear lamina (NL) preferentially interacts with inactive chromatin domains, referred to as heterochromatin. Externally, the NL serves as a platform to link the nucleus and the cytoskeleton via interaction with SUN and KASH domain proteins, referred to as the linker of nuclear and cytoskeleton (LINC) complex. The ability to anchor multiple protein complexes and chromatin domains has raised hypotheses that the NL plays critical roles in nuclear and cytoplasmic processes by maintaining the structural integrity of cytoskeleton and/or regulating transcription/signaling. The inheritance pattern of each gene is indicated. AD, autosomal dominant; AR, autosomal recessive; XL, X-linked; IU, inheritance unknown; INM, inner nuclear membrane; ONM, outer nuclear membrane; PNS, perinuclear space; NPC, nuclear pore complex.

sarcomere proteins such as cardiac actin (ACTC1), myosinbinding protein $\mathrm{C}$ (MYBPC3), $\alpha$-myosin heavy chain (MYH6), $\beta$ myosin heavy chain (MYH7), troponin $\mathrm{C}$ (TNNC1), troponin I (TNNI3), troponin T (TNNT2), $\alpha$-tropomyosin (TPM1), and titin (TTN) (Fatkin et al., 2014; Kamisago et al., 2000).

Among genes encoding these proteins, mutations in $\mathrm{MYH7}$ and TTN are most common in familial DCM cases (Fatkin et al., 2014; Sanbe, 2013). MYH7 encodes the $\beta$-myosin heavy chain (MHC- $\beta$ ), which is predominantly found in the heart and it forms the thick filament in the cardiac muscle together with other regulatory light chain proteins encoded by MYL2 and MYL3. The interaction between the head domains of MHC and cardiac actins provides the mechanical force required for cardiac muscle contraction. MYH7 mutations in residues S532 and F764, which are positioned in the actin binding and converter domains, respectively, were first reported as a cause of DCM (Kamisago et al., 2000). Moreover, an assay that utilizes myosin harvested from S532P and F764L mutant mice revealed that alterations in these residues lead to reduced performance of myosin, which in turn results in DCM through defective force generation in the process of sarcomere contraction (Debold et al., 2007; Schmitt et al., 2006). In other genetic screening, additional MYH7 mutations (I201T, T412N, A550V [head domain], T1019N, R1193S, $\mathrm{E} 1426 \mathrm{~K}$, and R1634S [tail domain]) were identified as causes of familial DCM (Villard et al., 2005). In all tested patients with these mutations, skeletal muscle defects were not detected, consistent with the predominant expression of $\mathrm{MYH7}$ in the heart.

Various titin isoforms form a separate filamentous system in the sarcomere; this system provides elasticity and structural integrity to the sarcomere. Titin isoforms range from 3.0 to 3.8 mega Daltons in molecular weight and are encoded by the single gene TTN, which consists of 363 exons (Bang et al., 2001). The $\mathrm{N}$-terminal domains of titin proteins from two adjacent sarcomeres are anchored at the Z-disc in an antiparallel fashion, overlapping with each other. Interactions of titin with Zdisc proteins (i.e., T-cap/telethonin and $\alpha$-actinin) are implicated in this intersarcomere linkage. The centrally positioned I-band 
region is responsible for the elasticity of titin filaments. The large isoformal variation within the I-band region of titin results in various titin filaments in different types of muscle. For example, the N2B domain is cardiac muscle-specific, whereas both skeletal and cardiac titin have the N2A domain. The C-terminal region of titin is connected to myosin filaments through binding to myosin and myosin-binding protein $\mathrm{C}$, which may account for the force transmission generated by ATP-driven myosin movement along the actin filaments during muscle contraction. Similar to the $\mathrm{N}$-terminal regions, the $\mathrm{C}$-terminal regions of titin from each side in a sarcomere juxtapose at the M-line. This overlapped positioning of titin at the $\mathrm{N}$ - and $\mathrm{C}$-terminal regions enables two sarcomeres to be physically linked and provides the myofibrils with mechanical elasticity. In 2002, mutations in titin were first identified as a cause of DCM (Itoh-Satoh et al., 2002). V54M and A743V mutations positioned in the Z-disc anchoring region of titin have been detected in Japanese patients with DCM. An in vitro assay revealed that these mutations reduced the binding affinity of titin to Z-disc proteins. In the same study, additional sequence variations were also detected in the N2B domain of titin. Although titin is considered to be a primary genetic factor associated with $\mathrm{DCM}$, the comprehensive study of its mutations has been hampered by the large size of the gene. Using advanced DNA sequencing technologies, a recent human genetic study revealed mutations in TTN in 72 of 312 DCM cases, with cosegregation observed in most affected families (Herman et al., 2012). Most of these sequence variations are nonsense, frame-shift, or splicing-site mutations, which are likely to generate truncated titin proteins. These mutations in titin are highly enriched in the $A$ band region, whereas no mutation has been found in the M-line and Z-disc regions. Therefore, truncation mutations in the $A$ band region of titin are most common in patients with DCM.

\section{DEFECTIVE STRUCTURAL PROTEINS CAUSING DCM}

Normal heart function requires faithful delivery of contractile force from the sarcomere to the plasma membrane (the sarcolemma for muscle cells) and the extracellular matrix. Multiple filamentous systems that physically connect the sarcomere to the sarcolemma are thought to play critical roles in force transmission (Fig. 1). Desmin, encoded by the DES gene, forms a filamentous system that links the nucleus, myofibrils, and plasma membrane. Desmin filaments surround the myofibrils at the Z-disc and attach to the sarcolemma via desmosome proteins, such as desmoplakin, plakoglobin, and plakophilin-2. Internally, desmin filaments are also connected to the nuclear envelope. These interactions result in bundling of adjacent myofibrils and anchor the myofibril bundle to the sarcolemma and the nuclear envelope, providing structural integrity to the sarcomere. In a candidate-gene approach, the $1451 \mathrm{M}$ mutation of the tail region of desmin was identified as a cause of DCM (Li et al., 1999) and found to cosegregate in a four-generation family with DCM. Affected family members had left ventricular dilation and heart failure, followed by sudden death. A mutation in the central rod domain of desmin was also found to be associated with skeletal muscle diseases; however, patients with DCM harboring the 1451M mutation did not exhibit clinically significant indications of skeletal muscle defects, suggesting that there are distinct molecular pathways underlying skeletal and cardiac myopathies. Consistent with this notion, desmin-null mice exhibit abnormal alignment of myofibrils in cardiomyocytes, leading to degeneration and necrosis of the myocardium (Milner et al., 1996).

The connections among sarcomeric actin, dystrophin, dys- trophin-associated proteins, and the extracellular matrix also play important roles in force transmission and provide structural integrity to the sarcolemma. Dystrophin is a 427-kDa rod-shape cytoplasmic protein. An actin-binding domain in the $\mathrm{N}$-terminus and dystroglycan complex-binding domain in the C-terminus are divided by a long central rod domain. Although mutations in dystrophin manifest as skeletal muscular dystrophy, affected individuals also exhibit DCM phenotypes. $D M D$, the single gene encoding dystrophin, is expanded 2.5 megabases on the $X$ chromosome. Most $D M D$ mutations are inherited in an $\mathrm{X}$-linked recessive manner. Duchenne muscular dystrophy (DMD) is the most prevalent disease resulting from mutations in $D M D$ and is characterized by gradual muscle weakness in young boys; female carriers rarely have symptoms upon $X$ chromosome inactivation. Patients with DMD often exhibit cardiac disorders and develop heart failure, mostly at the last stage of the disease. A few recent reports have shown that cardiovascular treatments improve the clinical status in patients with dystrophin muscular dystrophy (Kamdar and Garry, 2016). In several cases, mutations in $D M D$ caused $\mathrm{X}$-linked dilated cardiomyopathy (XLDCM) without clinical signs of skeletal muscle defects (Berko and Swift, 1987; Muntoni et al., 1993). XLDCM is characterized by a more rapid progression of cardiomyopathy compared with DMD and leads to the death of affected individuals at the age of $10-20$ years. A mouse experiment revealed that Coxsackievirus-mediated postnatal cleavage of dystrophin caused disassembly of the dystrophin complex and development of DCM in infected mice, suggesting that malfunction of dystrophin can cause acquired DCM (Badorff et al., 1999). The $\mathrm{N}$-terminus of dystrophin interacts with actin, and the Cterminus binds the extracellular matrix via the dystrophinassociated glycoprotein complex (DGC), which is a multiprotein complex spanning the plasma membrane. The DGC consists of $\alpha-, \beta-, \gamma$-, and $\delta$-sarcoglycans; $\alpha$ - and $\beta$-dystroglycans; syntrophin; DGC-associated proteins (DAPs); dystrobrevin; caveolin-3; and neuronal nitric oxide synthase (nNOS). Fragility of the sarcolemma caused by loss of DGC proteins is thought to cause DCM. Indeed, mutation in the SGCD gene encoding $\delta$-sarcoglycan leads to familial and sporadic DCM (Tsubata et al., 2000).

\section{$\mathrm{Ca}^{2+}$ CYCLING AND ION CHANNEL PROTEINS IN DCM}

Abnormal calcium homeostasis can also cause DCM and other heart diseases. $\mathrm{Ca}^{2+}$ enters cardiomyocytes through voltagegated calcium channels (LTCCs) embedded in the sarcolemma. Influx of $\mathrm{Ca}^{2+}$ triggers opening of the RyR calcium channel in the sarcoplasmic reticulum (SR), which initiates sarcomere contraction. Re-influx of released cytoplasmic $\mathrm{Ca}^{2+}$ into the SR to relax the sarcomere is mediated by the SR calcium-ATPase pump (SERCA2a). Phospholamban, encoded by the PLN gene, modulates $\mathrm{Ca}^{2+}$ recycling by inhibiting SERCA2a, and its activity is blocked by protein kinase A (PKA)-mediated phosphorylation. The R9C mutation in phospholamban causes DCM in a dominant-negative manner (Schmitt et al., 2003). A transgenic mouse experiment revealed that the $\mathrm{R} 9 \mathrm{C}$ mutation prevents PKA-mediated phosphorylation of phospholamban, leading to constitutive inhibition of SERCA2a and delaying uptake of cytoplasmic $\mathrm{Ca}^{2+}$. Therefore, abnormal intracellular $\mathrm{Ca}^{2+}$ cycling is thought to cause DCM in human patients with the R9C mutation.

Dysfunction of cardiac ion channel proteins appears to cause DCM. The SCN5A gene encodes a sodium channel subunit. SCN5A protein is predominantly found in the heart and it plays a critical role in the modulation of the heartbeat. DCM has been 
found to be caused by missense and deletion mutations in SCN5A (Olson et al., 2005). Patients with SCN5A mutations exhibit early-onset DCM with concomitant atrial fibrillation and conduction defects. The SUR2A subunit of the ATP-sensitive potassium channel in the heart, which is thought to adjust the membrane potential required for metabolic sensing of heart function. Mutations in the ABCC9 consists gene encoding SUR2A have been reported as causes of DCM (Bienengraeber et al., 2004).

\section{VARIATIONS IN NUCLEAR ENVELOPE PROTEINS LEADING TO DCM}

The nuclear envelope consists of inner and outer nuclear membranes, being divided by a perinuclear space (Fig. 1) (Stewart et al., 2007). The nuclear lamina is a protein meshwork located beneath the inner nuclear membrane (Butin-Israeli et al., 2012). Lamin proteins are the major components of the nuclear lamina. In mammals, three lamin genes, i.e. LMNB1, $\angle M N B 2$, and $L M N A$, encode all known lamin isoforms (Burke and Gerace, 1986; Gerace and Blobel, 1980). Emery-Dreifuss muscular dystrophy (EDMD) is a genetic disorder with joint contracture and progressive wasting and weakness of skeletal muscles (Emery, 2000). Many patients with EDMD also suffer from DCM and often exhibit conduction defects. Mutations in LMNA cause autosomal-dominant (AD-EDMD) and less frequent recessive (AR-EDMD) forms of EDMD (Bonne et al., 1999; Raffaele Di Barletta et al., 2000). LMNA mutations account for $5-8 \%$ of familial DCMs (Parks et al., 2008; Perrot et al., 2009; van Tintelen et al., 2007).

Mutations causing AD-EDMD identified by an initial genetic analysis include Q6X, R453W, R527P, and L530P; these mutations are enriched in the $\mathrm{C}$-terminal tail domain of lamin $\mathrm{A} / \mathrm{C}$ (Bonne et al., 1999). Later studies showed that AD-EDMD also results from $\mathrm{H} 222 \mathrm{Y}$ and $\mathrm{R} 249 \mathrm{Q}$ mutations (Bonne et al., 2000; Raffaele Di Barletta et al., 2000). A family study revealed that LMNA mutations also resulted in DCM without skeletal muscle disorders (Fatkin et al., 1999). In this study, 11 families with autosomal dominant DCM and conduction system failure were evaluated, and five novel $L M N A$ mutations were identified: R60G, L85R, N195K, E205G, and R571S. Each mutation caused inheritable conduction system disorders (atrial arrhythmia, atrioventricular conduction block, or sinus bradycardia) and DCM. Affected family members often exhibited heart failure and sudden death. However, no family member had clinical indications of EDMD, such as joint contracture or skeletal myopathy. Moreover, four of these five mutations are positioned in $\alpha$-helical rod domains, whereas the remaining mutation was detected in the C-terminal tail domain of lamin A/C (Fatkin et al., 1999). This result implies that structural alteration of the $\alpha$ helical domain of lamin A/C selectively causes DCM with conduction system disorders, whereas defects in the tail domain lead to diverse clinical features. Indeed, another mutation in the tail domain of LMNA (R482Q) was identified in patients with Dunnigan-type familial partial lipodystrophy (FPLD), which is associated with adipocyte defects, insulin resistance and diabetes, does not induce muscular or cardiac defects (Cao and Hegele, 2000).

$L m n a^{--}$mice are the first $L$ mna-mutant animal model created (Sullivan et al., 1999) and were established by removing the Cterminal regions encoded by exon 8 to the first half of exon 11 . $\mathrm{Lmna}^{--}$offspring resulting from intercross of $\mathrm{Lmna}^{+/}$mice undergo overtly normal embryonic development as compared to their heterozygous and wild-type littermates. However, their growth is significantly reduced starting from 2-3 weeks after birth, and they finally die at approximately 8 weeks of age, primarily because of muscle and cardiac defects. In a detailed study, DCM with conduction abnormality was developed by 4-6 weeks of age in $\mathrm{Lmna}^{-/}$mice (Nikolova et al., 2004), and the left ventricle (LV) of the heart was found to be dilated, round in shape, and thin-walled compared to that in wild-type mice. $\mathrm{Lmna}^{+/-}$mice show an intermediate severity of cardiac dysfunction and cellular defects in cardiomyocytes at 4-6 weeks of age. Interestingly, an independent study showed that $\mathrm{Lmna}^{+/}$mice develop severe cardiac abnormalities at 12 months of age analogous to those in humans with LMNA-mediated DCM (Wolf et al., 2008). Kubben et al. created the $L m n a^{G T / G T}$ mouse model to study loss of function of Lmna (Kubben et al., 2011). $L m n a^{G T / G T}$ mice were created by placing a gene trap cassette between exon $1 \mathrm{C}$ and exon 2 of Lmna, which introduced the Lmna exon 1- or exon 1C- $\beta$-geo fusion allele. Consequently, $L m n a^{G T / G T}$ mice did not produce any functional or dominantnegative variants of lamin A or lamin C protein. In contrast, $L m n a^{-/}$mice express a truncated protein from exon 1 to exon 7 that seemingly exerts weak toxic effects. $L m n a^{G T / G T}$ mice develop without overt abnormalities. Starting from postnatal day 7 , the growth of $L m n a^{G T / G T}$ mice is reduced, and growth ceases at postnatal day 13 . The mice then die at approximately 16-18 days of age, exhibiting defective postnatal development in multiple tissues; therefore, this is the mouse model closest to complete Lmna loss of function to date. Mouse models of missense LMNA mutations identified in patients with AD-EDMD have been also reported. $L m n a^{H 222 P / H 222 P}$ mice were created by introducing a point mutation into exon 4 of Lmna (Arimura et al., 2005), and they exhibit indications of muscular dystrophy and $\mathrm{DCM}$ at approximately 8 weeks after birth, which is analogous to the symptoms observed in humans with AD-EDMD. In another study, a transgenic mouse line that overexpresses Lmna M371K had a significantly lower birth rate than expected and typically died at 2-7 weeks after birth, which suggests that the M371K mutation in Lmna can recapitulate human disease in a dominant-negative manner. Immunohistochemical examination revealed that expression of the $\mathrm{M} 371 \mathrm{~K}$ isoform of lamin $\mathrm{A}$ in cardiomyocytes leads to distortion of nuclear shape, which may lead to tissue and organ disorder. Mounkes et al. (2005) generated Lmna ${ }^{N 195 K / N 195 K}$ mice, which harbor a missense mutation in exon 3 of Lmna. These mice develop DCMspecific phenotypes and eventually die from arrhythmia at 1216 weeks of age.

Mouse models of LMNA cardiomyopathy provide significant molecular insights into the pathophysiology of this disease. Gene expression profiling indicated that hearts from Lmna ${ }^{H 222 P / H 222 P}$ mice overexpressed downstream targets of the MAPK cascade such as ERK1/2 and JNK (Muchir et al., 2007). Treatment of $L m n a^{H 222 P / H 222 P}$ mice with the MEK inhibitor PD98059 significantly reduced cardiac defects as measured by normal cardiac function without overt LV dilation at 4 months of age (Muchir et al., 2009). Administration of the JNK inhibitor SP600125 to Lmna ${ }^{H 222 P / H 222 P}$ mice also alleviated cardiac defects (Wu et al., 2011). Studies using the $L m n a^{-/}$mouse model have proposed another target for treatment of LMNA cardiomyopathy. Recent studies showed the expression level of mTORC1 pathway components was increased in heart and skeletal muscles of $L m n a^{--}$mice (Choi et al., 2012; Ramos et al., 2012). Treatment of $L m n a^{--}$mice with rapamycin or temsirolimus, which are mTOR inhibitors, effectively blocked mTORC1 signaling, alleviated muscle dysfunctions, and prolonged the lifespan of these mice. 


\section{CONCLUSION}

$\mathrm{DCM}$ is a life-threatening disease that frequently results in heart failure. Identifying genetic factors that cause the clinical manifestation of a disease is required for defining the molecular trigger that initiates the disorder and the cellular response to the trigger. Moreover, complete profiling of the genetic causes of a disease is beneficial for an accurate diagnosis, particularly for diseases in which the clinical outcome is unclear or overlaps with other diseases. To date, genetic analyses of patients with familial DCM have shown causative relationships for mutations in more than 40 genes. These gene products are classified as sarcomere and structural proteins, ion channels, and nuclear envelope components. Profound insights into the molecular etiology of DCM have been provided by these discoveries. Failure of contractile force generation and transfer to the extracellular matrix, inefficient $\mathrm{Ca}^{2+}$ cycling, defective ion transport, and nuclear fragility can lead to dilation of ventricles and systolic dysfunction. Although current treatment of DCM is largely limited to the alleviation of clinical symptoms, further elucidation of these molecular processes and their cellular responses should provide new strategies to cure or mitigate cardiac dysfunction and heart failure. Moreover, monogenic genetic disorders such as familial DCM may be excellent targets for gene editing technology. The CRISPR/Cas9 system binds to the target sequence in the genome and generates double-stranded breaks with an unprecedented high efficiency. Mutated genes can be replaced by correct sequences via homology-directed repair (HDR). Indeed, the CRISPR/Cas9 system has been used to successfully correct dystrophin gene mutations in $\mathrm{mdx}$ mice. Therefore, this newly emerged technique is of great interest for therapies to treat human patients with familial DCM.

\section{ACKNOWLEDGMENTS}

We thank the members of the Kim laboratory for critical comments. This work was supported by the National Research Foundation of Korea (grant number: NRF-2014R1A1A1037106) and the Soonchunhyang University Research Fund. The authors declare that they have no conflicts of interest.

\section{REFERENCES}

Arimura, T., Helbling-Leclerc, A., Massart, C., Varnous, S., Niel, F., Lacene, E., Fromes, Y., Toussaint, M., Mura, A.M., Keller, D.I., et al. (2005). Mouse model carrying H222P-Lmna mutation develops muscular dystrophy and dilated cardiomyopathy similar to human striated muscle laminopathies. Hum. Mol. Genet. 14, 155-169.

Badorff, C., Lee, G.H., Lamphear, B.J., Martone, M.E., Campbell, K.P., Rhoads, R.E., and Knowlton, K.U. (1999). Enteroviral protease 2A cleaves dystrophin: evidence of cytoskeletal disruption in an acquired cardiomyopathy. Nat. Med. 5, 320-326.

Bang, M.L., Centner, T., Fornoff, F., Geach, A.J., Gotthardt, M., McNabb, M., Witt, C.C., Labeit, D., Gregorio, C.C., Granzier, H., et al. (2001). The complete gene sequence of titin, expression of an unusual approximately $700-\mathrm{kDa}$ titin isoform, and its interaction with obscurin identify a novel Z-disc to I-band linking system. Circ Res. 89, 1065-1072.

Berko, B.A., and Swift, M. (1987). X-linked dilated cardiomyopathy. N Engl. J. Med. 316, 1186-1191.

Bienengraeber, M., Olson, T.M., Selivanov, V.A., Kathmann, E.C., O'Cochlain, F., Gao, F., Karger, A.B., Ballew, J.D., Hodgson, D.M., Zingman, L.V., et al. (2004). ABCC9 mutations identified in human dilated cardiomyopathy disrupt catalytic KATP channel gating. Nat. Genet. 36, 382-387.

Bonne, G., Di Barletta, M.R., Varnous, S., Becane, H.M., Hammouda, E.H., Merlini, L., Muntoni, F., Greenberg, C.R., Gary F., Urtizberea, J.A., et al. (1999). Mutations in the gene encoding lamin A/C cause autosomal dominant Emery-Dreifuss muscular dystrophy. Nat. Genet. 21, 285-288.

Bonne, G., Mercuri, E., Muchir, A., Urtizberea, A., Becane, H.M. Recan, D., Merlini, L., Wehnert, M., Boor, R., Reuner, U., et al. (2000). Clinical and molecular genetic spectrum of autosomal dominant Emery-Dreifuss muscular dystrophy due to mutations of the lamin A/C gene. Ann. Neurol. 48, 170-180.

Burke, B., and Gerace, L. (1986). A cell free system to study reassembly of the nuclear envelope at the end of mitosis. Cell 44 , 639-652.

Butin-Israeli, V., Adam, S.A., Goldman, A.E., and Goldman, R.D. (2012). Nuclear lamin functions and disease. Trends Genet. 28, 464-471.

Cao, H., and Hegele, R.A. (2000). Nuclear lamin A/C R482Q mutation in canadian kindreds with Dunnigan-type familial partial lipodystrophy. Hum. Mol. Genet. 9, 109-112.

Choi, J.C., Muchir, A., Wu, W., Iwata, S., Homma, S., Morrow, J.P., and Worman, H.J. (2012). Temsirolimus activates autophagy and ameliorates cardiomyopathy caused by lamin $A / C$ gene mutation. Sci. Transl. Med. 4, 144ra102.

Codd, M.B., Sugrue, D.D., Gersh, B.J., and Melton, L.J., 3rd (1989) Epidemiology of idiopathic dilated and hypertrophic cardiomyopathy. A population-based study in Olmsted County, Minnesota 1975-1984. Circulation 80, 564-572.

Debold, E.P., Schmitt, J.P., Patlak, J.B., Beck, S.E., Moore, J.R., Seidman, J.G., Seidman, C., and Warshaw, D.M. (2007). Hypertrophic and dilated cardiomyopathy mutations differentially affect the molecular force generation of mouse alpha-cardiac myosin in the laser trap assay. Am. J. Physiol. Heart Circ. Physiol 293, H284-291.

Dellefave, L., and McNally, E.M. (2010). The genetics of dilated cardiomyopathy. Curr. Opin. Cardiol. 25, 198-204.

Emery, A.E. (2000). Emery-Dreifuss muscular dystrophy - a 40 year retrospective. Neuromuscul. Disord.10, 228-232.

Fatkin, D., MacRae, C., Sasaki, T., Wolff, M.R., Porcu, M., Frenneaux, M., Atherton, J., Vidaillet, H.J., Jr., Spudich, S., De Girolami, U., et al. (1999). Missense mutations in the rod domain of the lamin A/C gene as causes of dilated cardiomyopathy and conduction-system disease. N Engl. J. Med. 341, 1715-1724.

Fatkin, D., Seidman, C.E., and Seidman, J.G. (2014). Genetics and disease of ventricular muscle. Cold Spring Harb. Perspect. Med. 4, a021063

Gerace, L., and Blobel, G. (1980). The nuclear envelope lamina is reversibly depolymerized during mitosis. Cell 19, 277-287.

Herman, D.S., Lam, L., Taylor, M.R., Wang, L., Teekakirikul, P., Christodoulou, D., Conner, L., DePalma, S.R., McDonough, B. Sparks, E., et al. (2012). Truncations of titin causing dilated cardiomyopathy. N Engl. J. Med. 366, 619-628.

Itoh-Satoh, M., Hayashi, T., Nishi, H., Koga, Y., Arimura, T., Koyanagi, T., Takahashi, M., Hohda, S., Ueda, K., Nouchi, T., et al. (2002). Titin mutations as the molecular basis for dilated cardiomyopathy. Biochem. Biophys. Res. Commun. 291, 385393.

Kamdar, F., and Garry, D.J. (2016). Dystrophin-deficient cardiomyopathy. J. Am. Coll. Cardiol. 67, 2533-2546.

Kamisago, M., Sharma, S.D., DePalma, S.R., Solomon, S., Sharma P., McDonough, B., Smoot, L., Mullen, M.P., Woolf, P.K., Wigle, E.D., et al. (2000). Mutations in sarcomere protein genes as a cause of dilated cardiomyopathy. N Engl. J. Med. 343, 16881696.

Kubben, N., Voncken, J.W., Konings, G., van Weeghel, M., van den Hoogenhof, M.M., Gijbels, M., van Erk, A., Schoonderwoerd, K., van den Bosch, B., Dahlmans, V., et al. (2011). Post-natal myogenic and adipogenic developmental: defects and metabolic impairment upon loss of A-type lamins. Nucleus 2, 195-207.

Li, D., Tapscoft, T., Gonzalez, O., Burch, P.E., Quinones, M.A., Zoghbi, W.A., Hill, R., Bachinski, L.L., Mann, D.L., and Roberts, R. (1999). Desmin mutation responsible for idiopathic dilated cardiomyopathy. Circulation 100, 461-464.

Maron, B.J., Towbin, J.A., Thiene, G., Antzelevitch, C., Corrado, D., Arnett, D., Moss, A.J., Seidman, C.E., Young, J.B., American Heart, A., et al. (2006). Contemporary definitions and classification of the cardiomyopathies: an American Heart Association Scientific Statement from the Council on Clinical Cardiology, Heart Failure and Transplantation Committee; Quality of Care and Outcomes Research and Functional Genomics and Tran- 
slational Biology Interdisciplinary Working Groups; and Council on Epidemiology and Prevention. Circulation 113, 1807-1816.

Milner, D.J., Weitzer, G., Tran, D., Bradley, A., and Capetanaki, Y. (1996). Disruption of muscle architecture and myocardial degeneration in mice lacking desmin. J. Cell Biol. 134, 12551270.

Miura, K., Nakagawa, H., Morikawa, Y., Sasayama, S., Matsumori, A., Hasegawa, K., Ohno, Y., Tamakoshi, A., Kawamura, T., and Inaba, Y. (2002). Epidemiology of idiopathic cardiomyopathy in Japan: results from a nationwide survey. Heart 87, 126-130.

Morita, H., Seidman, J., and Seidman, C.E. (2005). Genetic causes of human heart failure. J. Clin. Invest. 115, 518-526.

Mortality, G.B.D., and Causes of Death, C. (2015). Global, regional, and national age-sex specific all-cause and cause-specific mortality for 240 causes of death, 1990-2013: a systematic analysis for the Global Burden of Disease Study 2013. Lancet 385, 117-171.

Muchir, A., Pavlidis, P., Decostre, V., Herron, A.J., Arimura, T., Bonne, G., and Worman, H.J. (2007). Activation of MAPK pathways links LMNA mutations to cardiomyopathy in EmeryDreifuss muscular dystrophy. The Journal of clinical investigation $117,1282-1293$

Muchir, A., Shan, J., Bonne, G., Lehnart, S.E., and Worman, H.J. (2009). Inhibition of extracellular signal-regulated kinase signaling to prevent cardiomyopathy caused by mutation in the gene encoding A-type lamins. Hum. Mol. Genet. 18, 241-247.

Muntoni, F., Cau, M., Ganau, A., Congiu, R., Arvedi, G., Mateddu, A., Marrosu, M.G., Cianchetti, C., Realdi, G., Cao, A., et al. (1993). Brief report: deletion of the dystrophin muscle-promoter region associated with X-linked dilated cardiomyopathy. $\mathrm{N}$ Engl. J. Med. 329, 921-925

Nikolova, V., Leimena, C., McMahon, A.C., Tan, J.C., Chandar, S., Jogia, D., Kesteven, S.H., Michalicek, J., Otway, R., Verheyen, F., et al. (2004). Defects in nuclear structure and function promote dilated cardiomyopathy in lamin A/C-deficient mice. J. Clin. Invest. 113, 357-369.

Olson, T.M., Michels, V.V., Ballew, J.D., Reyna, S.P., Karst, M.L., Herron, K.J., Horton, S.C., Rodeheffer, R.J., and Anderson, J.L. (2005). Sodium channel mutations and susceptibility to heart failure and atrial fibrillation. Jama 293, 447-454.

Parks, S.B., Kushner, J.D., Nauman, D., Burgess, D., Ludwigsen, S., Peterson, A., Li, D., Jakobs, P., Litt, M., Porter, C.B., et al. (2008). Lamin A/C mutation analysis in a cohort of 324 unrelated patients with idiopathic or familial dilated cardiomyopathy. Am. Heart J. 156, 161-169.

Perrot, A., Hussein, S., Ruppert, V., Schmidt, H.H., Wehnert, M.S., Duong, N.T., Posch, M.G., Panek, A., Dietz, R., Kindermann, I., et al. (2009). Identification of mutational hot spots in LMNA encoding lamin $\mathrm{A} / \mathrm{C}$ in patients with familial dilated cardiomyopathy. Basic Res. Cardiol. 104, 90-99.

Raffaele Di Barletta, M., Ricci, E., Galluzzi, G., Tonali, P., Mora, M. Morandi, L., Romorini, A., Voit, T., Orstavik, K.H., Merlini, L., et al.
(2000). Different mutations in the LMNA gene cause autosomal dominant and autosomal recessive Emery-Dreifuss muscular dystrophy. Am. J. Hum. Genet. 66, 1407-1412.

Ramos, F.J., Chen, S.C., Garelick, M.G., Dai, D.F., Liao, C.Y., Schreiber, K.H., MacKay, V.L., An, E.H., Strong, R., Ladiges, W.C., et al. (2012). Rapamycin reverses elevated mTORC1 signaling in lamin A/C-deficient mice, rescues cardiac and skeletal muscle function, and extends survival. Sci. Transl. Med. 4, 144 ra103.

Sanbe, A. (2013). Dilated cardiomyopathy: a disease of the myocardium. Biol. Pharm. Bull. 36, 18-22.

Schmitt, J.P., Kamisago, M., Asahi, M., Li, G.H., Ahmad, F., Mende, U., Kranias, E.G., MacLennan, D.H., Seidman, J.G., and Seidman, C.E. (2003). Dilated cardiomyopathy and heart failure caused by a mutation in phospholamban. Science 299, 14101413.

Schmitt, J.P., Debold, E.P., Ahmad, F., Armstrong, A., Frederico, A. Conner, D.A., Mende, U., Lohse, M.J., Warshaw, D., Seidman, C.E., et al. (2006). Cardiac myosin missense mutations cause dilated cardiomyopathy in mouse models and depress molecular motor function. Proc. Natl. Acad. Sci. USA 103, 14525-14530.

Stewart, C.L., Roux, K.J., and Burke, B. (2007). Blurring the boundary: the nuclear envelope extends its reach. Science 318 , 1408-1412.

Sullivan, T., Escalante-Alcalde, D., Bhatt, H., Anver, M., Bhat, N., Nagashima, K., Stewart, C.L., and Burke, B. (1999). Loss of Atype lamin expression compromises nuclear envelope integrity leading to muscular dystrophy. J. Cell Biol. 147, 913-920.

Tsubata, S., Bowles, K.R., Vatta, M., Zintz, C., Titus, J., Muhonen, L., Bowles, N.E., and Towbin, J.A. (2000). Mutations in the human delta-sarcoglycan gene in familial and sporadic dilated cardiomyopathy. J. Clin. Invest. 106, 655-662.

van Tintelen, J.P., Hofstra, R.M., Katerberg, H., Rossenbacker, T. Wiesfeld, A.C., du Marchie Sarvaas, G.J., Wilde, A.A., van Langen, I.M., Nannenberg, E.A., van der Kooi, A.J., et al. (2007). High yield of LMNA mutations in patients with dilated cardiomyopathy and/or conduction disease referred to cardiogenetics outpatient clinics. Am. Heart J. 154, 1130-1139.

Villard, E., Duboscq-Bidot, L., Charron, P., Benaiche, A., Conraads, V., Sylvius, N., and Komajda, M. (2005). Mutation screening in dilated cardiomyopathy: prominent role of the beta myosin heavy chain gene. Eur. Heart J. 26, 794-803.

Wolf, C.M., Wang, L., Alcalai, R., Pizard, A., Burgon, P.G., Ahmad, F., Sherwood, M., Branco, D.M., Wakimoto, H., Fishman, G.I., et al. (2008). Lamin A/C haploinsufficiency causes dilated cardiomyopathy and apoptosis-triggered cardiac conduction system disease. J. Mol. Cell. Cardiol. 44, 293-303.

Wu, W., Muchir, A., Shan, J., Bonne, G., and Worman, H.J. (2011). Mitogen-activated protein kinase inhibitors improve heart function and prevent fibrosis in cardiomyopathy caused by mutation in lamin A/C gene. Circulation 123, 53-61. 